\title{
Perinatal and cardiovascular outcomes in a pregnant patient with Marfan syndrome
}

\author{
Maciej Osinski ${ }^{1}$, Stefan Ozegowski ${ }^{2}$, Jakub Kornacki ${ }^{1}$, Marta Kuciara ${ }^{3}$, \\ Malgorzata Szczepanska ${ }^{1}$, Ewa Wender-Ozegowska ${ }^{1}$ \\ 'Poznan University of Medical Sciences Division of Reproduction, Poland \\ ${ }^{2} 2$ nd Department of Cardiology, Poznan University of Medical Sciences, Poland \\ ${ }^{3}$ Poznan University of Medical Sciences, Poland
}

A 25-year-old primigravida with Marfan syndrome (MFS) was admitted at 36 weeks of gestation (WOG).

\section{History}

The patient was diagnosed with MFS at puberty and the syndrome was confirmed genetically. At the age of 20 she suffered from pneumothorax which was treated with thoracoscopic drainage. At the time of the patient's admission to our clinic, we specifically asked whether there had been any occurrence of cardiovascular-related symptoms prior to her pregnancy, and the patient denied there had been any such symptoms. A cervical pessary was used between the 24th and 32nd WOG. Intravenous tocolytic therapy for prolonging gestation and intramuscular corticosteroids for accelerating fetal lung maturation were both administered in the 32 nd WOG due to significant risk of a preterm birth. In the 2nd trimester of pregnancy, transthoracic echocardiogram (TTE) testing revealed an increased aortic maximal root dimension compared with the before-pregnancy TTE value ( 40 vs $44 \mathrm{~mm}$ ); and we observed no pathological changes in other echocardiographic parameters. During hospitalization at the 34th WOG, ECG results were normal and the TTE results were: maximal aortic root dimension $=46 \mathrm{~mm}$, and ascending aorta dimension $=33 \mathrm{~mm}$. The patient's sister was also diagnosed with MFS and suffered from aortic valve disease which had required surgical replacement.

\section{On physical examination}

The patient had an above-average height $(193 \mathrm{~cm})$, long limbs, arachnodactyly, a regular cardiac function, 80 BPM with auscultatory events properly accentuated, $\mathrm{BP}=80 / 50$, symmetrical radial pulse, meagre varicose veins on her lower extremities, and a shortened 1-centimeter dilated cervix.

\section{Cardiac assessment}

ECG results were normal (sinus rhythm, $80 \mathrm{bpm}$ ). No signs of progression were observed in TTE values (maximal aortic root dimension: $46 \mathrm{~mm}$ (Fig. 1), and ascending aorta dimension: $32 \mathrm{~mm}$ ). Due to Marfan syndrome being compli-

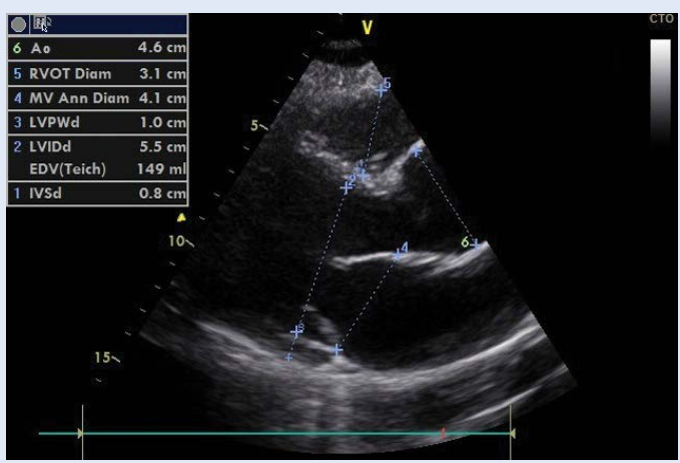

Figure 1 Parasternal long axis view — aorta root echocardiographic assessment prior to delivery

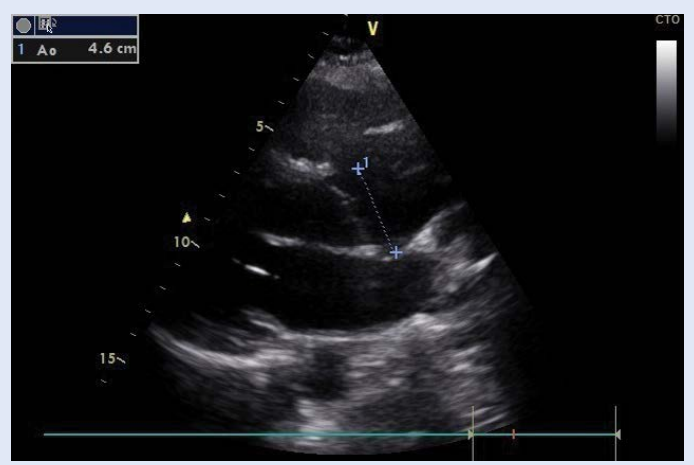

Figure 2 Parasternal long axis view — aorta root echocardiographic assessment after delivery

Corresponding author:

Maciej Osinski

Poznan University of Medical Sciences Division of Reproduction, Poland

e-mail: maciejosinski@me.com 
cated by little-altered maximal aorta root and ascending aorta dimensions after observation, we performed an elective caesarean delivery at term (newborn: healthy daughter, $3300 \mathrm{~g}, \mathrm{Ap} \mathrm{10,10).} \mathrm{During} \mathrm{the} \mathrm{early} \mathrm{puerperium} \mathrm{period,} \mathrm{the}$ patient did not report any cardiovascular-related symptoms and TTE results did not significantly differ from the last assessment made during the pregnancy (Fig. 2). During the puerperium period, low molecular weight heparin was used as a thromboprophylaxis, and 4 days after caesarean delivery the patient was discharged from hospital in good health.

Marfan syndrome remains the dominant autosomal genetic disease that affects connective tissue. The cardiovascular system, the skeleton and organs such as the pleura are all affected and therefore pneumothorax may occur. MFS prevalence is estimated at 1:5.000-1:6.500. Aortic dissection remains the major cause of mortality and morbidity in MFS patients, determined at $1.2 \%$ and $7.9 \%$, respectively. In the course of pregnancy, the risk of aortic dissection rises; and Tsuritani et al also described two such patients in their study. We conclude that it is important, when caring for women with MFS, to obtain a diagnosis prior to pregnancy. In cases of women not being diagnosed prior to pregnancy, the risk of life-threatening MFS complications during pregnancy, delivery or the puerperium period increases. Therefore, pregnancy in MFS women should be planned, and it is crucial to conduct careful echocardiographic assessments prior to pregnancy. For women with aortic diameters of $\geq 40 \mathrm{~mm}$, prophylactic aortic surgery is advised prior to pregnancy. However, the European Society of Cardiology (ESC) recommends surgery should always be performed prior to pregnancy in cases of a root aorta diameter of $>45 \mathrm{~mm}$. The association between MFS and risk of premature labour and delivery is well documented; and this was also demonstrated in our case. 